\title{
Guías metodológicas para levantamientos geodésicos en México
}

\author{
Javier Arellano Sánchez*
}

Recibido el 09 de mayo de 2016; aceptado el 12 de septiembre de 2016

\begin{abstract}
Geodetic data capture, revision, processing, analysis and integration anywhere in the world are directed to opportunely produce quality inputs and products under reference frames and proved standards in order to be useful in any spatial data infrastructure. To this effect, it is essential to use methodologies with steps that guide the implementation of effective methods and working procedures to reach the expected objectives. According to the above the methodological guides used in the National Institute of Statistics and Geography (INEGI) in order to support the National Subsystem of Geographic and Environmental Information are described in this article.
\end{abstract}

Key words: Methodological Guides, Geodetic data, Reference Frames, Standards, Spatial data, Mexico.

\section{Resumo}

A captação, a revisão, o processamento, a análise e a integração de dados geodésicos em qualquer lugar do mundo se orientam a gerar insumos e produtos com qualidade e oportunidade conforme marcos de referência e padrões comprovados de modo que sejam úteis para qualquier infraestrutura de dados espaciais, para isso é essencial o uso de metodologias com etapas que orientem a execução de métodos e procedimentos de trabalho efetivos para alcançar os objetivos traçados. Em função do anterior, o presente artigo descreve as guias metodológicas utilizadas no Instituto Nacional de Estatística e Geografia (INEGI) para dar suporte ao marco de referência geodésico do Subsistema Nacional de Informação Geográfica e do Meio Ambiente.

* Dirección General de Geografía y Medio Ambiente, Instituto Nacional de Estadística y Geografía (INEGI), Av. Héroe de Nacozari Sur 2301, Fraccionamiento Jardines del Parque, C.P. 20276, Aguascalientes, México, correo electrónico: javier.arellano@inegi.org.mx 
Palavras chave: Guias metodológicos, Dados Geodésicos, Marcos de Referência, Padrões, Dados Espaciais, México.

\section{Resumen}

La captación, la revisión, el procesamiento, el análisis y la integración de datos geodésicos en cualquier lugar del mundo se orientan a generar insumos y productos con calidad y oportunidad bajo marcos de referencia y estándares comprobados de modo que sean útiles para cualquier infraestructura de datos espaciales, para ello es esencial el uso de metodologías con etapas que orienten la ejecución de métodos y procedimientos de trabajo efectivos para alcanzar los objetivos trazados. En función de lo anterior, el presente artículo describe las guías metodológicas utilizadas en el Instituto Nacional de Estadística y Geografía (INEGI) para dar soporte al marco de referencia geodésico del Subsistema Nacional de Información Geográfica y del Medio Ambiente.

Palabras clave: Guías metodológicas, Datos Geodésicos, Marcos de Referencia, Estándares, Datos Espaciales, México.

\section{Generalidades}

En México, dentro del Sistema Nacional de Información Estadística y Geográfica, coordinado por el INEGI, se generan datos del Marco de Referencia Geodésico para contribuir al componente geográfico del Subsistema Nacional de Información Geográfica y del Medio Ambiente también denominado Infraestructura de Datos Espaciales de México; asimismo, éstos datos, son insumos para los procesos relacionados con la elaboración de la cartografía nacional, catastros, georreferencia de información de interés nacional, entre otros proyectos.

Como parte de las actividades del INEGI que permiten apoyar la capacitación y actualización de servidores públicos, así como en la investigación permanente en temas de producción y análisis de la información, se desarrollan documentos normativos y operativos, entre ellos metodologías para normalizar la captación, procesamiento, integración y difusión de datos, productos y servicios bajo estándares de calidad.

Las metodologías aplicadas a los levantamientos geodésicos generan datos e información que son puestos a disposición de los usuarios de manera gratuita a través de la red geodésica nacional, con el objeto de vincular sus estudios geográficos. Estos levantamientos al igual que otros, que se realizan en el Instituto en materia estadística y geográfica, llevan implícito el objetivo de suministrar a la sociedad y al Estado información de calidad, pertinente, veraz y oportuna, así como normar la generación de información estadística y geográfica en el marco del Sistema Nacional de Información Estadística y Geográfica (SNIEG). 


\section{Normatividad base para el diseño de guías metodológicas para levantamientos geodésicos en México}

Las disposiciones normativas, elaboradas en el INEGI, se orienta a regular el diseño, captación, producción, actualización, organización, procesamiento, integración y compilación de la información estadística y geográfica. La normatividad base para colectar y producir datos geodésicos e integrarlos al Sistema Nacional de Información Estadística y Geográfica consiste en:

- La Ley del Sistema Nacional de Información Estadística y Geográfica (LSNIEG)

- Reglamento Interno del INEGI (RII)

- Normas Técnicas: del Sistema Geodésico Nacional (NTSGN), Estándares de Exactitud Posicional (NTEEP) y sobre Elaboración de Metadatos Geográficos (NTEMG).

- El Diccionario de Datos Geodésicos.

La información geográfica es considerada como el conjunto organizado de datos espaciales georreferenciados, que mediante símbolos y códigos genera el conocimiento acerca de las condiciones físico-ambientales, de los recursos naturales y de las obras de naturaleza antrópica del territorio nacional.

Adicional a la Ley, el Instituto ejerce sus atribuciones y facultades de conformidad con la forma y términos establecidos en Reglamento Interno que para tal efecto aprobó su Junta de Gobierno. Dentro de sus atribuciones están la de realizar estudios en materia geográfica y cartográfica, que tenga por objeto la elaboración y actualización de la normatividad y la generación e integración de información geográfica, de acuerdo a las mejores prácticas internacionales; coadyuvar en la construcción del Subsistema de Información Geográfica y del Medio Ambiente y regular la producción de información geográfica del país, así como promover la investigación y desarrollo en la materia.

En materia de geografía y medio ambiente debe integrar y actualizar la información proveniente de los siguientes temas geográficos: marco de referencia geodésico; límites costeros, límites internacionales, límites estatales y municipales; datos de relieve continental, insular y submarino; datos catastrales y topográficos, de recursos naturales y clima, así como nombres geográficos.

La Norma Técnica del Sistema Geodésico Nacional contiene las disposiciones mínimas que definen el Sistema Geodésico Nacional, a partir de las cuales es posible integrar datos e información geodésica generada por las unidades del estado, a fin de establecer las condiciones necesarias para que el Marco Geodésico sea homogéneo, compatible, comparable y útil para la generación de información geográfica debidamente georreferida. 
La Norma Técnica de Estándares de Exactitud Posicional establece las disposiciones mínimas referentes a los valores de exactitud posicional para diferentes tipos de rasgos geográficos que deberán adoptarse para todo trabajo de referenciación ubicado dentro del Territorio Nacional, realizado por el Instituto y las Unidades del Estado que integran el Sistema Nacional de Información Estadística y Geográfica, ya sea por sí mismas o por terceros, así como promover su armonización y homogeneidad.

La Norma Técnica sobre Elaboración de Metadatos Geográficos establece las disposiciones mínimas para la elaboración de metadatos de los grupos de datos geográficos de interés nacional o que sirvan para generar estos, realizados por las Unidades del Estado que integran el Sistema, ya sea por sí mismas o por terceros, así como promover su armonización y homogeneidad.

El Diccionario de Datos Geodésicos muestra la forma en que la información geodésica producida en el INEGI se ha estructurado y descrito conceptualmente para poder ser ingresada a la Base de Datos Geográficos. Es un documento complementario a las normas técnicas en materia geográfica para regular la producción de datos espaciales y su integración. Contiene los nombres, definiciones y las características de los objetos espaciales que descritos bajo especificaciones comunes dan lugar a la generación de datos espaciales.

\section{Los levantamientos geodésicos, su importancia y utilidad}

En México, el papel más relevante de la geodesia consiste en el establecimiento, operación y mantenimiento de la Red Geodésica Nacional (RGN), constituida por puntos cuyas coordenadas se han obtenido por medio de diversos levantamientos integrados de tal forma que relacionan todas las porciones del territorio nacional en una sola estructura. Es decir, el principal uso está en integrar una red unificada que sea el marco fundamental de referencia para elaborar la cartografía de México, determinar la forma, ubicación y tamaño del país, así como de sus recursos naturales y obras de infraestructura que se asientan en éste, entre otras utilidades.

En el INEGI los levantamientos geodésicos están unidos a sistemas y marcos de referencia para la determinación de coordenadas de puntos convenientemente elegidos y situados en, sobre o cerca de la superficie terrestre, así como datos de altura y de gravedad que sirven para el desarrollo sostenible de su marco de referencia geodésico.

El campo gravitacional de la Tierra está sujeto a variaciones que se producen en el tiempo y en el espacio debido a una combinación de factores, tales como la distribución desigual de la masa en los océanos, los continentes, y el interior profundo, las variables relacionadas con el clima, como el balance hídrico de los continentes, el derretimiento de los glaciares o en crecimiento, así como los diferentes tipos de rocas con distintas densidades que originan las variaciones espaciales de la grave- 
dad. La variación de la gravedad es lo que se necesita medir en el tiempo (Watkins, 2002).

\section{Estructura del Marco Geodésico}

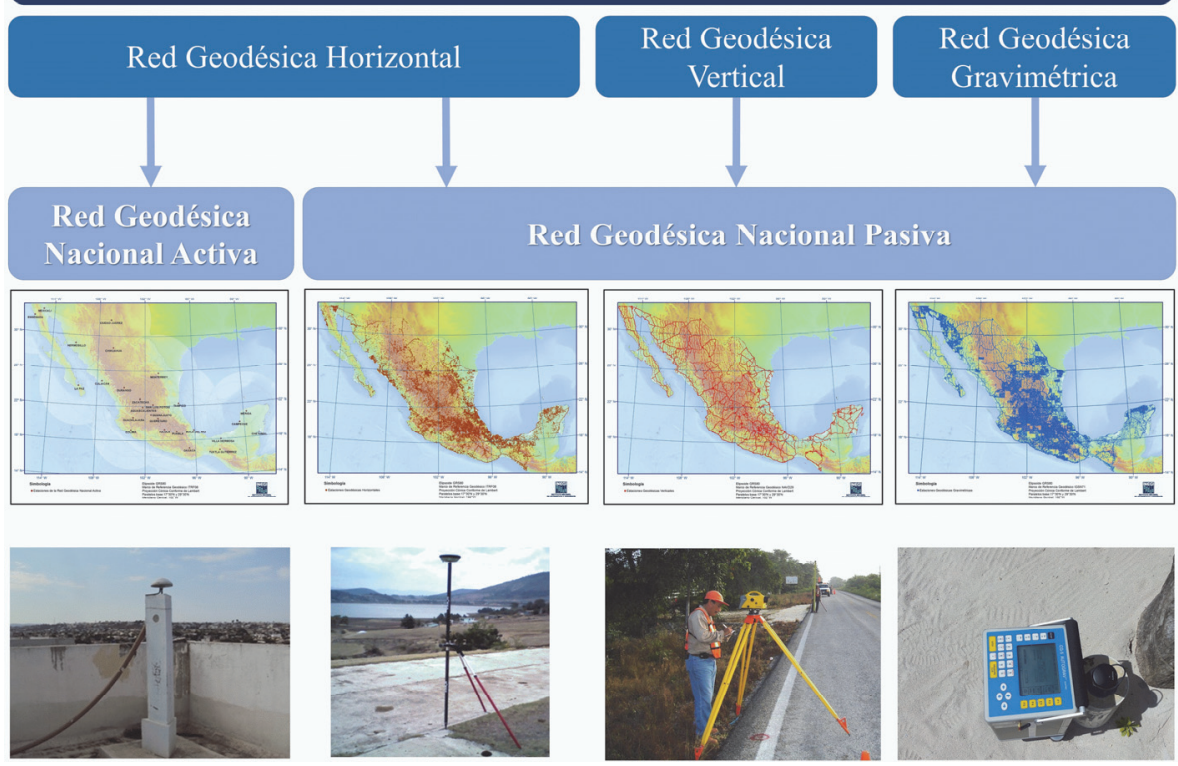

Figura 1. Marco de Referencia Geodésico.

Fuente: INEGI.

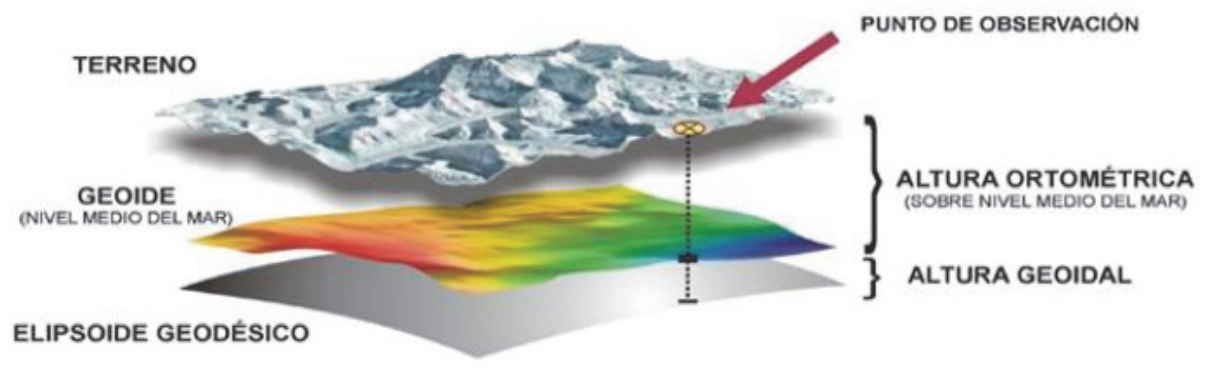

Figura 2. Determinación de coordenadas de puntos de observación. 
Los levantamientos geodésicos se realizan bajo lo señalado en las guías metodológicas elaboradas por el INEGI dan forma a un conjunto de procedimientos y operaciones de campo y gabinete, orientadas a dotar de valores geodésicos a puntos de observación particular sobre el terreno. Combinando información de un modelo de alturas geoidales (generadas a partir de un modelo geoidal) con alturas geodésicas (elipsoide de referencia) obtenidas mediante técnicas de posicionamiento satelital para calcular alturas ortométricas de cualquier punto de observación sobre el terreno (Figura 2).

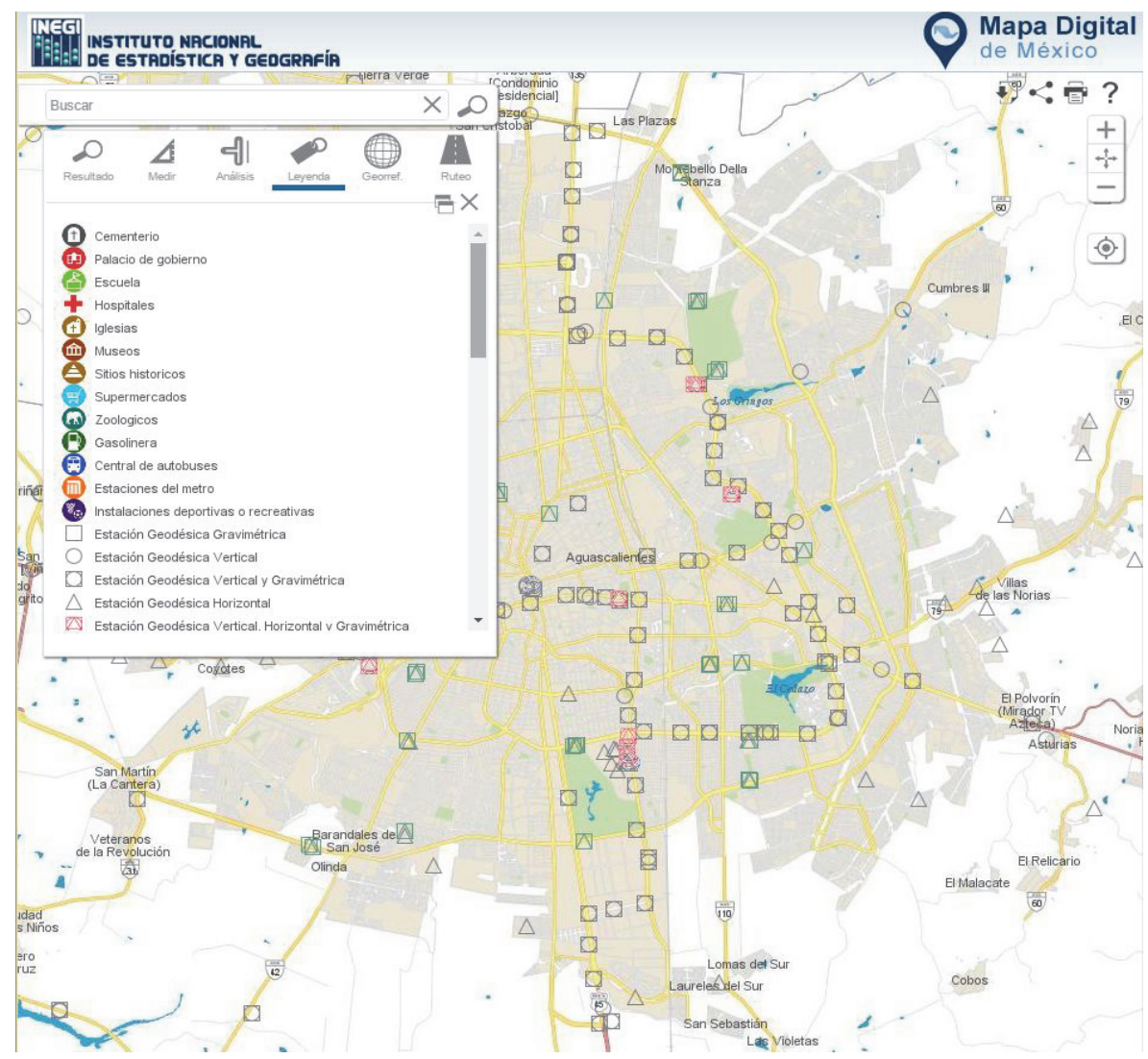

Figura 3. Datos geodésicos a través de servicios WEB.

Fuente: INEGI.

$<$ http://gaia.inegi.org.mx/mdm6/?v=bGF0OjIzLjMyMDA4LGxvbjotM AyLjE0NTY1LHo6MSxsOmMxMTFzZXJ2aWNpb3N8dGMxMTF zZXJ2aWNpb3M=> 

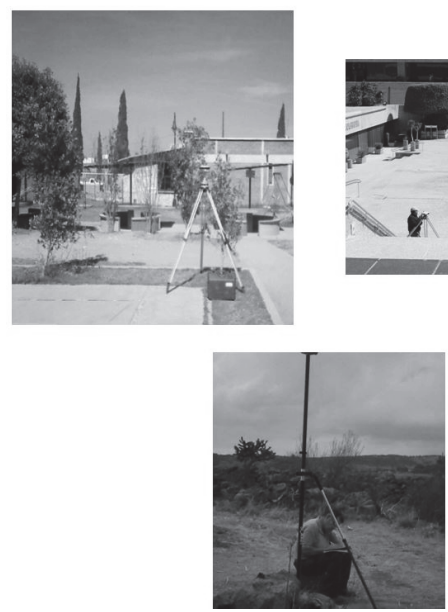
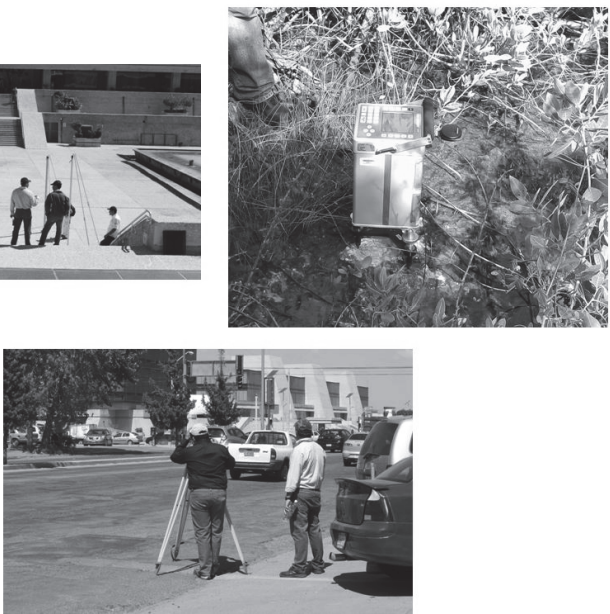

Figura 4. Levantamientos Geodésicos para la RGNP. Fuente: INEGI.

Los datos obtenidos son integrados en una base de datos y se ponen a disposición a los usuarios, de manera gratuita, en Internet mediante la solución geomática conocida como Mapa Digital de México (Figura 3), de este modo dar atención oportuna de datos geodésicos a las necesidades actuales y futuras de datos geodésicos a nivel nacional y aquellos que por acuerdos y convenios internacionales se tengan que compartir. Fuente: INEGI

Dentro del INEGI, los datos obtenidos mediante levantamientos geodésicos (Figura 4) conforman la Red Geodésica Nacional Pasiva, y se aplican para contribuir a la georreferencia de información de interés nacional, cómo la carta topográfica en la escala 1:50 0000, también para el estudio del comportamiento del territorio en la interrelación que guardan los componentes de potencial gravimétrico, desplazamiento horizontales y verticales; así como para estudios de investigación y para la planeación y diseño de obras culturales de diversa índole.

El uso de esta información brinda beneficios en cuanto al intercambio de información entre las diversas instituciones que ejercen las políticas públicas. Su cobertura es nacional y de actualización permanente.

Los Sistemas de Referencia de la Red Geodésica Nacional Pasiva (RGNP), para cada tipo de red, son actualmente los siguientes:

- Red Geodésica Horizontal: estaciones con coordenadas generadas a partir de levantamientos utilizando tecnología GPS/GNSS. Sistema de Referencia ITRF08 (International Terrestrial Reference Frame 2008, por sus siglas en inglés) época 2010.0, en el elipsoide GRS80 (Moritz, 2000). 


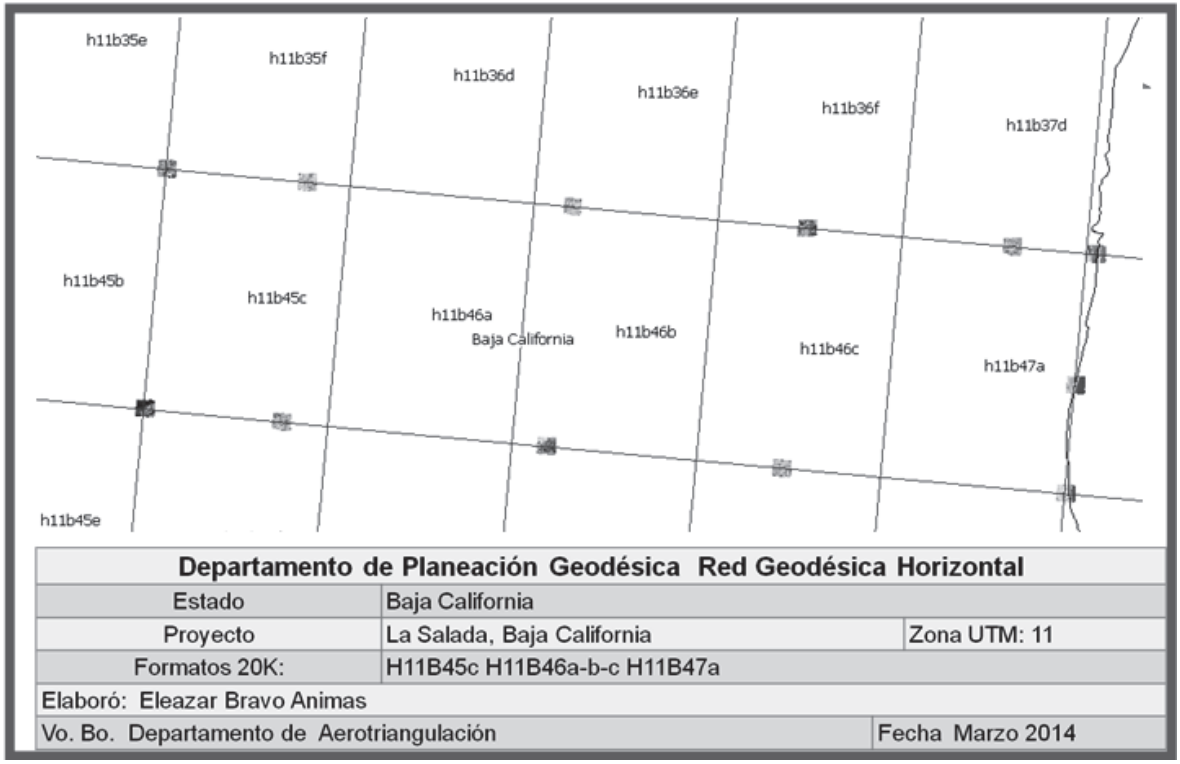

Figura 5. Proyecto de medición de Puntos de Control Terrestre. Fuente INEGI.

- Red Geodésica Vertical: estaciones en el terreno con altura o elevación con respecto al NAVD88 (North American Vertical Datum of 1988, por sus siglas en inglés), para el caso de México.

- Red Geodésica Gravimétrica: estaciones en el terreno con mediciones de valores del campo de gravedad de la tierra asociadas al Dátum IGSN71 (International Gravity Standardization Net of 1971, por sus siglas en inglés). Existe un gran número de ellas materializadas físicamente.

\section{Guías metodológicas}

Cada guía contiene apartados generales, un marco conceptual y la descripción metodológica de trabajo en cada red. A continuación se detalla de manera general las etapas de cada guía; para una mayor profundización se recomienda consultar las metodologías completas para cada tipo de red geodésica en el vínculo de Internet señalado:

\section{Guía Metodológica de la Red Geodésica Horizontal (RGH)}

La Guía Metodológica de la Red Geodésica Horizontal es utilizada por el Instituto en los levantamientos de campo necesarios para el establecimiento de Estaciones Geodésicas Horizontales, con procedimientos estandarizados y acordes con la nor- 
matividad vigente, lo que permite la compatibilidad y comparabilidad de la información para su integración al sistema. Los valores obtenidos son de posición con respecto al elipsoide de referencia, es decir latitud y longitud geodésicas, así como una altura elipsoidal y constituye una de las tres vertientes del Sistema Geodésico Nacional.

Los levantamientos geodésicos horizontales pueden incluir o no el establecimiento de marcas físicas identificadas con una placa metálica alojada en monumentos o empotradas sobre rocas u obras de infraestructura; su permanencia y estabilidad son factores de gran importancia para que los usuarios puedan vincular sus proyectos a la Red Geodésica Horizontal. En caso de realizar la monumentación de una placa, deberá seguir el procedimiento y especificaciones contenidas en la metodología de trabajo.

Dentro de los levantamientos en los que no se establecen marcas físicas, la ubicación de las posiciones en campo se hace con apoyo de imágenes de percepción remota; su importancia radica en utilizarse como insumos para el establecimiento de Puntos de Control Terrestre (PCT) ${ }^{1}$ que se aplican en el proceso fotogramétrico de triangulación, que a su vez facilita la elaboración de ortoimágenes; así como para la georreferenciación de servicios para el Marco Geoestadístico Nacional y Unidades Económicas del Directorio Estadístico Nacional de Unidades Económicas (DENUE), entre muchas aplicaciones más.

\section{Etapas}

\section{Planeación}

En esta etapa se revisan los recursos financieros y metas a lograr, insumos de trabajo, especificaciones del usuario en cuanto a cobertura, densidad y exactitud posicional. Asimismo se revisan las condiciones de accesibilidad a la zona de trabajo, vías de comunicación, localidades y cualesquier otro elemento que facilite el logro del proyecto. Además es esencial la revisión del equipo y correcta operación, revisión y verificación de herramientas y accesorios, etcétera.

\section{Elaboración}

A partir de la definición de un programa de trabajo, se elaboran los proyectos de campo con la finalidad de obtener y proveer datos e información geodésica útiles

Ubicación representada mediante un croquis que correlaciona la ubicación de las coordenadas obtenidas con GNSS/GPS con respecto a una imagen de percepción remota; la ubicación gráfica y las coordenadas obtenidas tienen la finalidad de servir como insumo para el proceso fotogramétrico de triangulación aérea. 
para georreferir objetos espaciales y fenómenos de interés de acuerdo a la naturaleza específica de cada proyecto.

En los trabajos de apoyo a la elaboración de productos cartográficos se establecen Puntos de Control Terrestre considerando control histórico existente, traslape entre imágenes, colindancia con cuerpos de agua marítimos, formatos cartográficos ya controlados en las adyacencias del proyecto.

\section{Actividades de campo}

Dentro de las actividades de campo se realiza la localización y posicionamiento geodésico de los puntos donde se ubicarán y medirán Estaciones Geodésicas de densificación, así como el mantenimiento de la Red Geodésica Nacional, redes geodésicas de densificación, medición de monumentos fronterizos, Puntos de Control Terrestre para procesos fotogramétricos o Puntos de Apoyo Terrestre para actividades catastrales, entre otros.

De acuerdo al estándar de exactitud posicional, recursos disponibles, área de cobertura y facilidades de acceso se define el método y configuraciones de medición con equipos GPS/GNSS.

Los formatos de apoyo son muy importantes y sirven entre otros aspectos para describir la ubicación del punto medido en campo en un rasgo identificable, cuidando que los objetos vistos en el terreno corresponden con los vistos en la imagen. Se debe realizar un listado de los puntos observados para llevar un efectivo control.

Es esencial la elaboración de un croquis de la ubicación de la estación geodésica o punto de control con la información suficiente para coadyuvar con el proceso de triangulación o bien para integrar los datos a la Red Geodésica Nacional con base en los atributos del diccionario de datos.

El levantamiento de campo incluye el traslado al sitio de medición, selección final de cada sitio en donde se obtendrá el posicionamiento geodésico conforme al proyecto y condiciones del terreno, visibilidad satelital y la correcta correlación de punto elegido con su representación en la imagen de percepción remota. Una vez que se ubica el equipo y se programa la sesión de trabajo de acuerdo al método de medición predefinido, se va llenando una hoja de registro de observaciones GPS/GNSS.

\section{Procesamiento de los datos}

Consiste en la obtención de vectores y ajuste de coordenadas ligadas a la Red Geodésica Nacional Activa (RGNA) o Red Geodésica Nacional Pasiva (RGNP).

La liga a la RGNP se hace sobre estaciones horizontales establecidas físicamente en el terreno mediante una mojonera y placa metálica que identifica su ubicación tridimensional respecto a un marco de referencia para una determinada época y es 
susceptible de actualizar sus valores conforme al requerimiento de un proyecto determinado. En caso necesario, se deberá realizar la conversión de los datos GPS/GNNS nativos a formato RINEX (Receiver Independent Exchange Format), así como tener los datos de las estaciones de referencia y la información levantada en el registro de observaciones de campo; asimismo, en su caso, descargar las efemérides precisas según corresponda a las especificaciones de exactitud posicional. Teniendo todos los insumos y el proyecto dentro del software de procesamiento geodésico con todos los parámetros configurados se realiza el procesamiento diferencial.

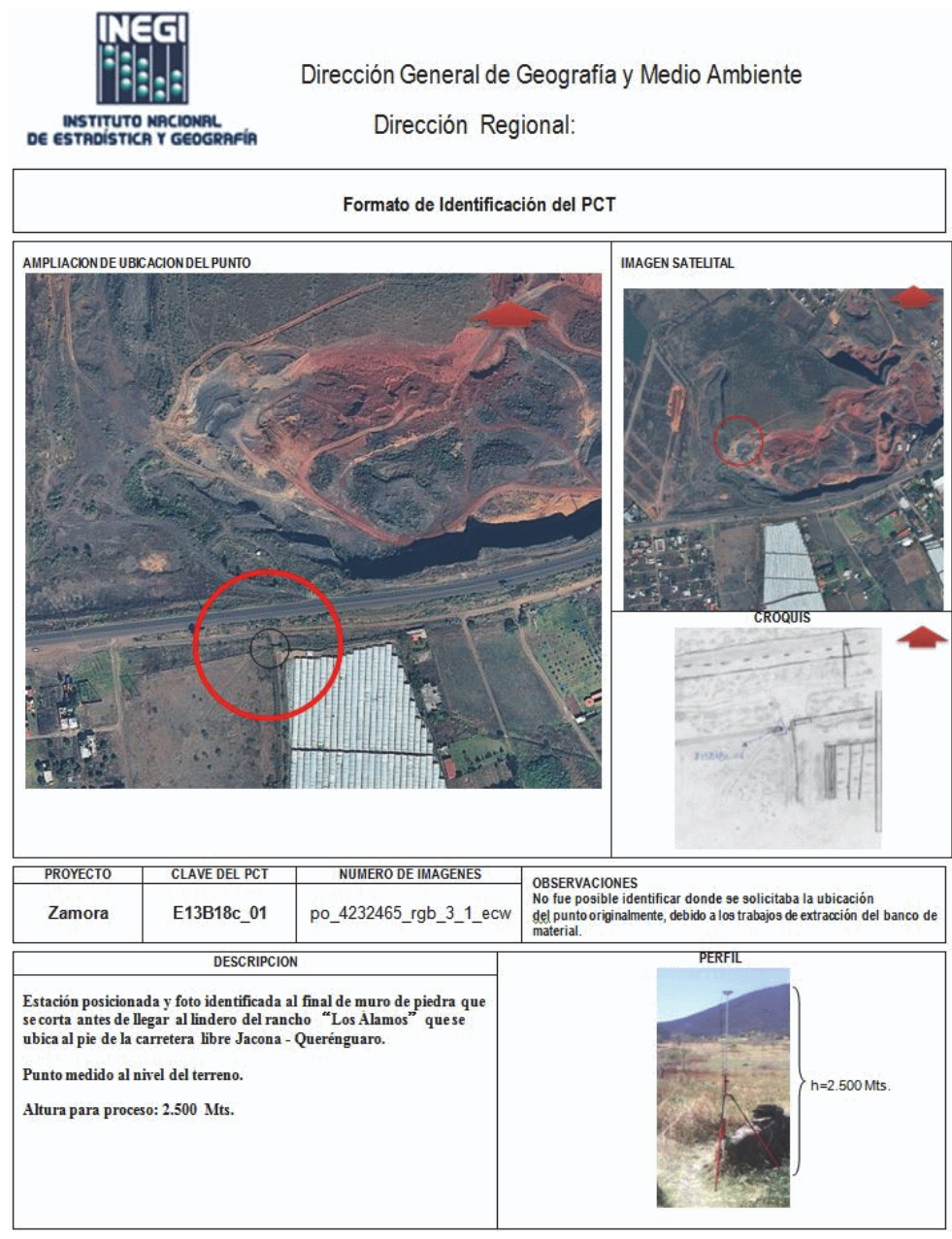

Figura 6. Formato de Identificación de un Punto de Control Terrestre.

Fuente: INEGI. 


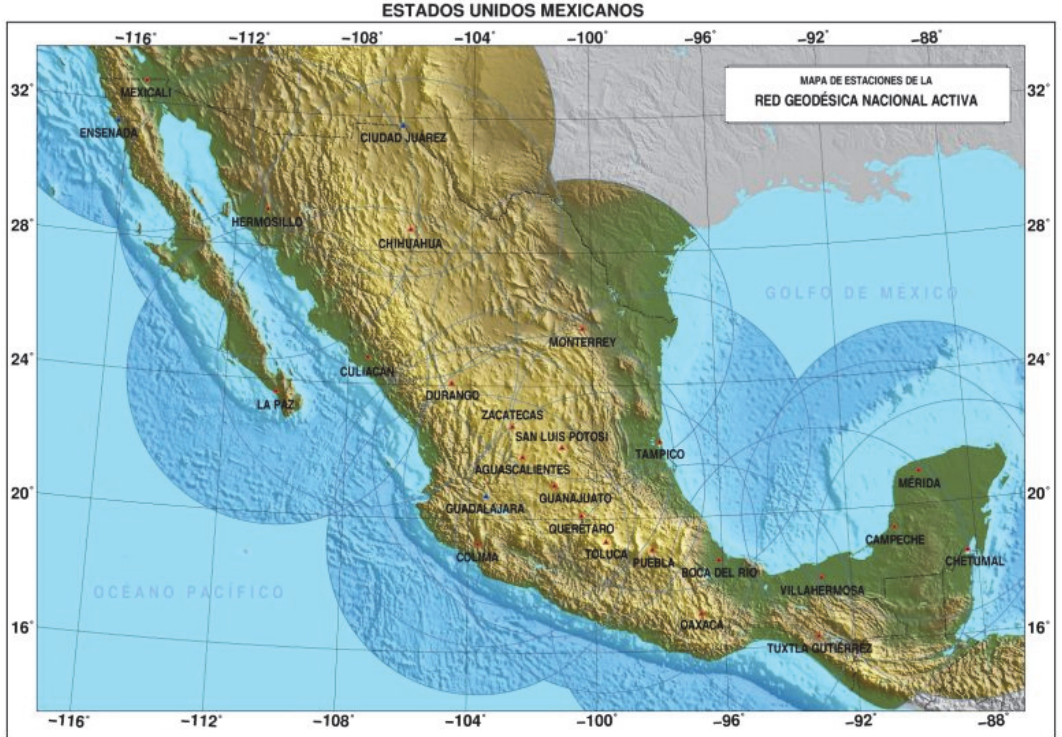

Figura 7. Red Geodésica Nacional Activa.

Fuente: INEGI.

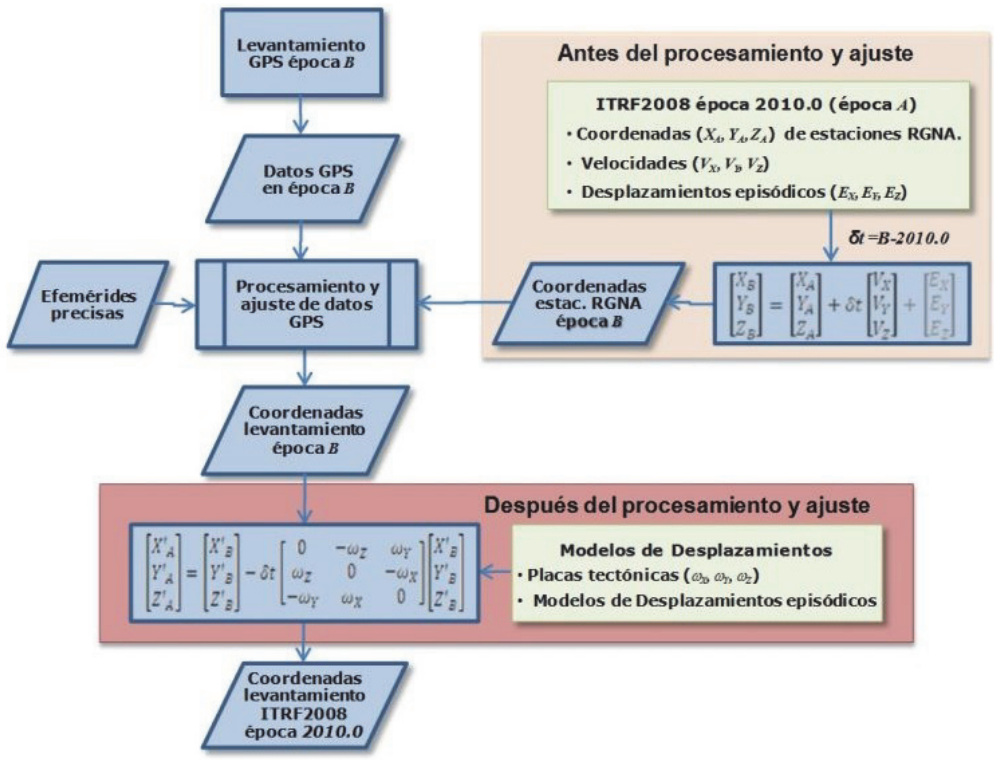

Figura 8. Procedimiento general para el procesamiento de datos GPS/GNSS considerando deformaciones del marco geodésico en el tiempo.

Fuente: INEGI. 
El procesamiento cuando se tiene una exigencia muy alta de exactitud posicional deberá realizarse con método riguroso (Figura 8), el cual incluirá el uso de modelos de placas, las velocidades de las estaciones de referencia y datos sobre desplazamientos episódicos; con ello se podrá trasladar la ubicación de las estaciones geodésicas o puntos de control terrestre a diferentes épocas dentro del mismo marco de referencia. Los elementos para el procesamiento riguroso con GNSS/GPS son.

Coordenadas ITRF2008 época 2010.0 con alta exactitud de las CORS (Estaciones de Referencia de Operación Continua, por sus siglas en inglés) y las velocidades de las estaciones de la RGNA.

- Modelos de placas

- Desplazamientos episódicos ${ }^{2}$

Para las estaciones de referencia (RGNA o CORS) se tienen:

- Velocidades $\left(\mathrm{V}_{\mathrm{X}}, \mathrm{V}_{\mathrm{Y}}, \mathrm{V}_{\mathrm{Z}}\right)$

- Desplazamientos episódicos $\left(\mathrm{E}_{\mathrm{X}}, \mathrm{E}_{\mathrm{Y}}, \mathrm{E}_{\mathrm{Z}}\right)$

Para el resto del territorio se tienen:

- Modelos de velocidades de placas tectónicas ( $\left.\mathrm{w}_{\mathrm{X}}, \mathrm{w}_{\mathrm{Y}}, \mathrm{w}_{\mathrm{Z}}\right)$

- Modelos de desplazamientos episódicos ${ }^{3}$

Estos elementos nos permiten trasladar la ubicación de puntos a diferentes épocas (dentro de un mismo marco de referencia).

\section{Validación}

Consiste en verificar que los resultados de los levantamientos de PCT con GPS/GNSS, mediante la revisión cualitativa y cuantitativa de las especificaciones señaladas en el proyecto, para asegurar su entrega con calidad a las áreas usuarias.

En esta etapa el personal que realiza la validación deberá conocer los diferentes procedimientos, parámetros utilizados y la normatividad vigente. Asimismo deberá registrar en una bitácora los datos e información que le servirá para elaborar el

2 Eventos sísmicos que afectan regiones específicas. Los registros de desplazamientos en la zona afectada permiten generar un modelo de desplazamientos.

3 Solo se considera su uso en el caso en que alguna estación tuvo algún desplazamiento local por sismo que deba corregirse con $\left(E_{X}, E_{Y}, E_{Z}\right)$, y que en el área del punto o los puntos a procesar no están ubicados en una zona que haya tenido desplazamientos episódicos (por sismo). La zona de los puntos medidos también puede estar afectados localmente por algún sismo y debieran corregirse también. 
reporte final del proyecto conforme a las especificaciones contenidas en la correspondiente guía metodológica.

\section{Guía Metodológica de la Red Geodésica Vertical (RGV)}

La Red Geodésica Vertical constituye una de las tres vertientes del Sistema Geodésico Nacional y está representada por un conjunto de puntos situados sobre la superficie del Territorio Nacional. Cada punto fue establecido sobre una marca física (placa en un monumento o empotrada) para obtener su valor de elevación o altura determinada por posicionamiento geodésico vertical con respecto a un nivel de referencia.

El Posicionamiento Geodésico Vertical consiste en determinar la elevación de puntos convenientemente elegidos y demarcados en el terreno, sobre una superficie de referencia, para el caso de México se está migrando de NAV29 a NAVD88. Estos puntos se integran en redes de Posicionamiento Geodésico Vertical. El propósito del Posicionamiento Geodésico Vertical es crear el marco de referencia nacional, que sirva para el control de elevaciones mediante el establecimiento, densificación y mantenimiento de la Red Geodésica Vertical.

\section{Etapas}

\section{Planeación}

En esta etapa de realiza la recopilación de insumos útiles para realizar el anteproyecto y levantamientos de campo conforme a metas y recursos asignados anualmente. El anteproyecto facilita el conocimiento amplio de la zona de trabajo, además de preseleccionar los posibles sitios para establecer nuevas Estaciones Geodésicas Verticales (EGV) o Bancos de Nivel (BN) tomando en cuenta que la separación entre éstos sea acorde a las especificaciones requeridas para el proyecto; se puede considerar una distancia aproximada de $2 \mathrm{~km}$, entre cada Banco de Nivel, exceptuando aquellas que se realicen como ramales a las localidades dentro del proyecto, densificaciones, tramos dentro de las poblaciones, zonas montañosas con pendientes fuertes y zonas costeras, en donde se podrán establecer de manera aproximada a cada kilómetro. En caso de realizar mediciones para determinar redes mareográficas (BN que se requieran establecer cercanos a mareógrafos) se podrán ubicar a una distancia aproximada de $300 \mathrm{~m}$.

En el proyecto preliminar se incluyen los posibles bancos de liga, los nuevos $\mathrm{BN}$ proyectados $\mathrm{y}$, en su caso, los $\mathrm{BN}$ de alguna Red Mareográfica (de existir), incluyendo el banco maestro.

Para establecer control geodésico vertical en localidades urbanas que permita, entre otras utilidades, dar apoyo a estudios de subsidencia se deberá privilegiar la medición sobre circuitos que circunden el área urbana, separaciones entre bancos no 
mayores a $1,000 \mathrm{~m}$ ni menores a $500 \mathrm{~m}$, entre otros aspectos señalados en la guía metodológica.

\section{Reconocimiento, verificación y proyecto definitivo}

El reconocimiento estará a cargo del responsable de la brigada y tendrá como objetivo verificar en campo el anteproyecto, con el fin de realizar las modificaciones necesarias para el proyecto definitivo. Dichas modificaciones pudieran ser ocasionadas por accidentes naturales o artificiales que sólo son detectables por observación directa.

Dentro de las actividades en esta etapa están la selección del lugar para establecer el nuevo BN, considerando su permanencia y estabilidad, que ofrezca, en lo posible, condiciones de medición con GPS/GNSS, entre otros aspectos señalados en la guía metodológica.

\section{Monumentación}

El establecimiento de marcas físicas que representen a los BN podrá consistir en:

a) Placa ahogada en monumento de concreto

b) Placa empotrada en roca sana

c) Placa empotrada verticalmente sobre un paramento

d) Placa empotrada en obra de concreto o estructura arquitectónica

En la etapa de monumentación se elabora un croquis de ubicación de la EGV o $\mathrm{BN}$ y se describen atributos de acuerdo con el Diccionario de Datos Geodésicos y aquellos para el análisis y procesamiento de la información. La actualización del croquis, descripciones o itinerarios de las marcas geodésicas deberá realizarse cada vez que se lleve a cabo la recuperación y medición de líneas de nivelación o bien se visite la EGV o BN para realizar otro tipo de mediciones geodésicas o simplemente como parte de la recuperación y mantenimiento de la Red Geodésica Nacional Pasiva.

\section{Levantamiento}

Se deberá tener previamente preparado todo el equipamiento, accesorios y herramientas de trabajo y llevar a cabo la comprobación y ajustes necesarios del equipo de medición antes del levantamiento. De igual manera se deberá verificar la configuración de coeficientes y rango de parámetros como distancia mínima de una visual, alturas mínima y máxima de observaciones, parámetros de registro y generales del equipo de acuerdo a las especificaciones del fabricante. 
La nivelación de cada sección se hace bajo condiciones atmosféricas diferentes para dirección de ida y de vuelta e independiente una de la otra, cumpliendo con la tolerancia establecida. En caso de no cumplir con dicha tolerancia, la nivelación debe repetirse tantas veces como sea necesario hasta que las dos nivelaciones en direcciones opuestas cumplan con la tolerancia.

La comprobación de estabilidad entre BN se puede establecer hasta en dos secciones consecutivas, secciones de uno o dos $\mathrm{km}$; si la primera no cumple con la tolerancia y ambas si lo cumplen, esto significa que el BN intermedio tuvo alguna perturbación.

El método recomendable de observación en cada puesta de aparato puede ser: alternado ADAD/DADA (atrás-adelante-atrás-adelante/adelante-atrás-adelanteatrás). Esto asegura que siempre se estará observando a la misma mira o estadal, al iniciar las mediciones en cada puesta de aparato. El método alternado es muy útil para realizar nivelación geodésica que requiere alta precisión a diferencia de la nivelación diferencial topográfica que precisión, con el método alternado se logra una mejor distribución del error por curvatura.

Se puede realizar cualesquier otro método siempre y cuando se garanticen los estándares de exactitud posicional y tolerancias admisibles.

La discrepancia de una sección debe estar dentro de los límites de la tolerancia establecida en función de la distancia mínima de la sección y la especificación vigente. Se debe tomar en cuenta que existen varias correcciones, por ejemplo la corrección ortométrica, que afectan directamente los valores de los desniveles, los que en el proceso de la información deberán ser reportados por el programa de ajuste como fuera de tolerancia, si fuera el caso; por lo tanto es altamente recomendable considerar no trabajar en campo al límite de dichas tolerancias y llevar el control de la discrepancia acumulada para determinar, en su caso, cual o cuales secciones habrá que repetirse.

Diariamente, antes de iniciar los trabajos de nivelación, se realiza la determinación del error de colimación del nivel (determinación de "C").

Asimismo se deberán hacer los cálculos necesarios y el llenado de formatos específicos relacionados con las actividades de campo, lo anterior con la finalidad de detectar un posible error y solucionarlo en la misma etapa de medición.

Cada día, dentro del periodo de comisión, al finalizar los trabajos de nivelación y dependiendo del equipo de medición utilizado, es recomendable que el personal que forme parte de la brigada de campo efectúe los cálculos de desniveles, distancias, diferencias estadimétricas, etcétera, de cada sección y la codificación de éstos y otros datos, como temperaturas y fechas.

Una vez concluidos los trabajos parciales de campo de una línea o circuito de nivelación, se procederá a la codificación digital de cada uno de los formatos espe- 
cíficos, para que al término de los mismos se cuente con la información lista para el proceso y análisis de resultados, así como del respaldo de la información.

Las anomalías, omisiones y errores cometidos en estos periodos parciales deben ser atendidas de inmediato en el mismo periodo de trabajo.

El resumen de la línea consiste en un formato que puede incluir además de la información observada en campo la distancia acumulada, las discrepancias, así como información de temperatura, posición geográfica, valor de "C", entre otros. Dicha información es de sumo interés debido a que son el insumo principal para llevar a cabo el proceso de ajuste de los datos.

El archivo de entrada para el proceso se estructura con la información de la línea; se tiene a la mano el valor máximo admisible de discrepancia para una sección, datos del equipo de medición, los datos del levantamiento correspondientes a fechas, desniveles, factor de colimación, etcétera. Finalmente se estructuran los datos de acuerdo al software de proceso a utilizar.

\section{Proceso y análisis}

El proceso y ajuste de los datos debe realizarse utilizando el software compatible con los datos colectados por la Unidad del Estado responsable del levantamiento, de acuerdo al tipo de Nivel utilizado, y debe ser capaz de aplicar como mínimo el método de mínimos cuadrados para el ajuste de los datos.

Para realizar el análisis se verifica la información del archivo de resultados, los cuales deben estar estructurados incluyendo las siguientes secciones:

- Resumen de datos

- Resumen de Bancos de Nivel

- Resumen de los Bancos de Nivel fijos

- Corrección Ortométrica aplicada

- Cálculo del desnivel promedio y rechazo de observaciones

- Tabla de ligas

- Varianza, covariancia, matriz de pesos, diferencia de alturas, etcétera

- Elevaciones ajustadas

- Resumen de secciones y desniveles

El desnivel Ortométrico (Figura 9 se obtiene mediante la suma de corrección ortométrica, más desnivel geométrico de cada sección que componen las líneas de nivelación).

La Corrección Ortométrica que se aplica en el INEGI, actualmente, se basa en la siguiente fórmula: 
$\mathrm{OC}_{\mathrm{AB}}=\frac{\bar{g}_{A B}-G}{G} \Delta L_{A B}+H_{A}^{O} \frac{\bar{g}_{A}^{\prime}-G}{G}-H_{B}^{O} \frac{\bar{g}_{B}^{\prime}-G}{G}$

Donde:

OC Corrección ortométrica

$g_{A}, g_{B} \quad$ Valores de gravedad sobre el terreno del punto A y B respectivamente

$\bar{g}_{A B} \quad$ Gravedad media entre los banco A y B en mGal

$\Delta L_{A B} \quad$ Desnivel geométrico entre los bancos A y B en metros

$H_{A}^{O}, H_{B}^{O} \quad$ Altura ortométrica aproximada de A y B en metros $\bar{g}_{A}^{\prime}, \bar{g}_{B}^{\prime} \quad$ Valor medio de la gravedad a lo largo de la línea de plomada de A

$\bar{g}^{\prime}{ }_{A}=g_{A}+0.0424 \frac{m G a l}{m} H_{A}^{o}$

$\bar{g}_{B}^{\prime}=g_{B}+0.0424 \frac{m G a l}{m} H_{B}^{o}$

$\mathrm{G}=980619.943 \mathrm{mGal}$, que corresponde al modelo de gravedad sintético en latitud 45.0 y altura geodésica de 0.0 metros.

Las elevaciones ajustadas son aceptadas en función de la ausencia de errores, anomalías o inconsistencias y teniendo como referencia los estándares de exactitud posicional vigentes.

Por último se integra el expediente de la línea o circuito nivelado que incluye entre otros elementos el croquis de ruta de la nivelación, respaldo de observaciones, formatos, archivos de datos, croquis con descripciones de cada Banco de Nivel.

\section{Guía Metodológica de la Red Geodésica Gravimétrica}

La Red Geodésica Gravimétrica constituye una de las tres vertientes del Sistema Geodésico Nacional. Está representada por el conjunto de puntos con valores de gravedad obtenidos a través de Levantamientos Gravimétricos que comprenden la medida de valores absolutos o relativos del valor de la gravedad sobre puntos situados en la superficie terrestre. Su propósito fundamental es determinar el campo gravimétrico existente y su relación e influencia con los tipos de levantamiento geodésico horizontal y vertical. 


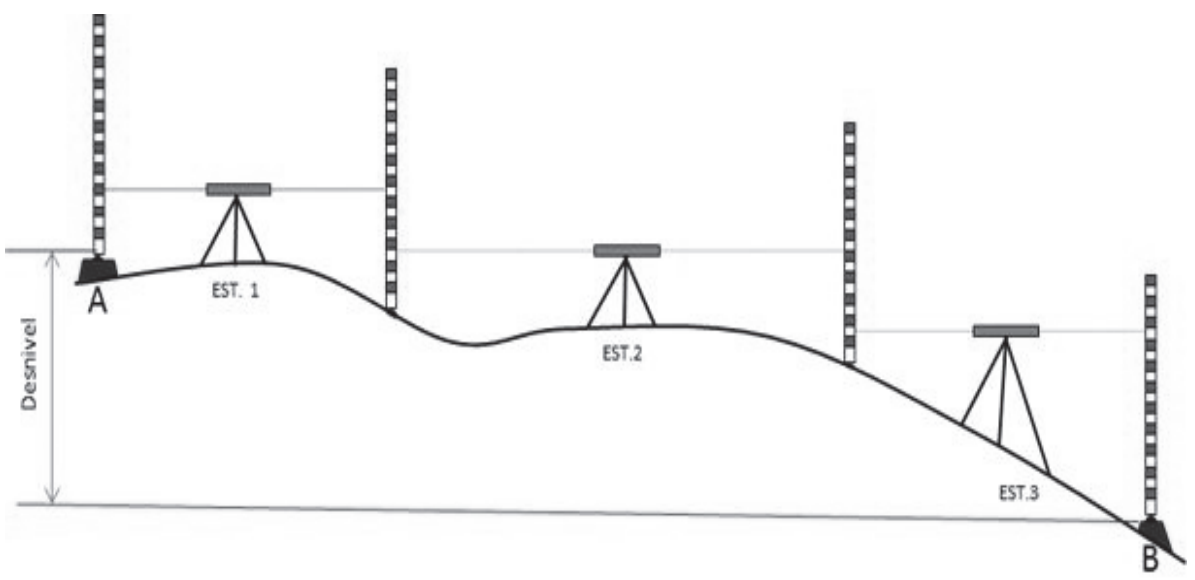

Figura 9. Desnivel Ortométrico.

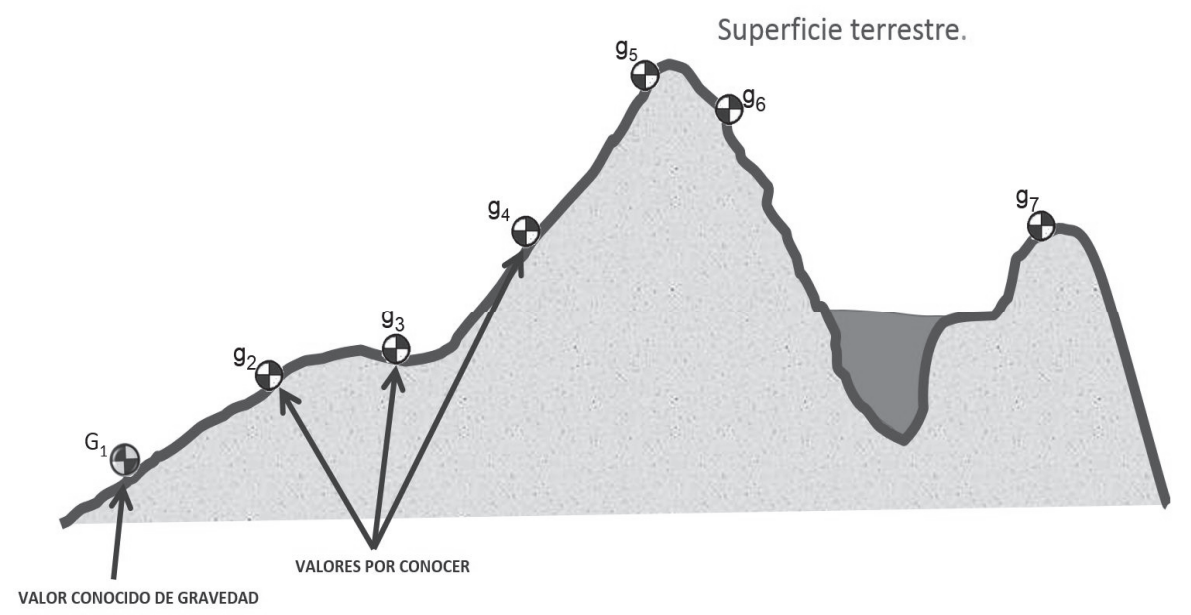

Figura 10. Determinación gravimétrica relativa.

Fuente: INEGI.

Los métodos de levantamiento de gravedad dependen directamente de los instrumentos disponibles para hacer las observaciones.

La guía metodológica describe el establecimiento de estaciones base de gravedad (primer orden) y estaciones geodésicas gravimétricas (segundo orden), para conocer la distribución del campo gravimétrico externo.

Es el insumo básico para llegar al conocimiento del geoide en México con el que, entre otras utilidades, se puede conocer la relación con el elipsoide. 


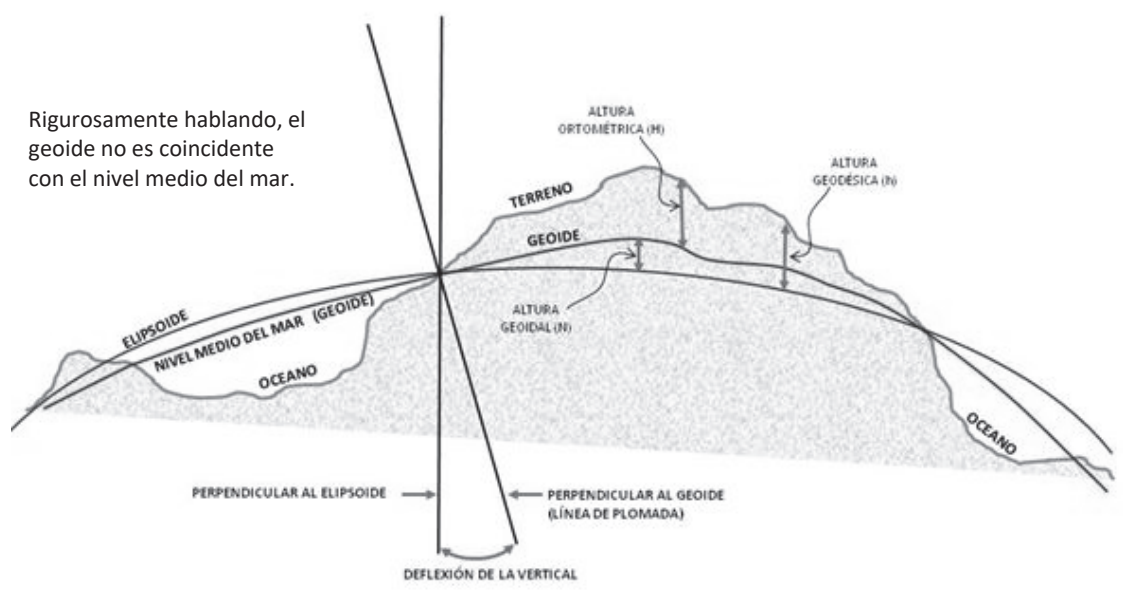

Figura 11. Geoide y sus superficies relacionadas.

Fuente: INEGI.

\section{Etapas}

\section{Planeación}

En la planeación se deben considerar primeramente los recursos necesarios: humanos, financieros y materiales, que permitan cubrir operativos que incluyan seguridad, profesionalismo y aplicación de tecnologías, para alcanzar las metas propuestas.

\section{Elaboración del proyecto}

Consiste en el acopio de insumos necesarios y suficientes para los trabajos de medición de gravedad terrestre.

\section{Definición de la zona de trabajo}

Con apoyo de cartografía impresa o digital de la zona geográfica del proyecto es posible identificar la zona proyectada para realizar los trabajos gravimétricos, la ubicación de las Estaciones Base de Gravedad (EBG), las estaciones gravimétricas regionales con marca física sobre bancos de nivel y vértices geodésicos que serán útiles para determinar los trabajos de campo.

\section{Verificación del gravímetro}

Verificar y controlar el factor de escala del gravímetro. Por lo que el aparato se debe exponer, en lo posible, a cambios de gravedad conocidos en una línea de cali- 
bración, para detectar una variación, y de ser necesario realizar su ajuste. Es recomendable verificar este factor por lo menos una vez al año.

Previamente al levantamiento se deben de verificar aspectos importantes de los gravímetros tales como parámetros iniciales, temperatura interna, carga de baterías, cables, plato base o trípode, navegador GPS, altímetro, cargadores de corriente alterna y directa, etcétera.

Las mediciones relativas de gravedad se realizarán con liga a estaciones de primer orden conocidas como Estaciones Base de Gravedad (EBG), en el mejor de los casos con liga a una estación de gravedad absoluta.

Las EBG se consideran como estaciones de primer orden ligadas al marco de referencia IGSN71 para apoyar la densificación de valores de gravedad (estaciones de segundo orden) y son el insumo principal de Geoide Gravimétrico Mexicano (GGM).

\section{Medición en campo}

Para estaciones Gravimétricas Base o Línea de Calibración:

Los criterios para la selección de un sitio para el establecimiento de una estación base o línea de calibración para la densificación de valores de gravedad de segundo orden consisten en asegurar su permanencia, condiciones de estabilidad, accesibilidad, espacio para colocar el equipo de medición e aislamiento. Se debe hacer un croquis y formato de descripción para futuras medidas o recuperación de la estación.

En las operaciones de la Red Geodésica Gravimétrica, al igual que en las otras vertientes, se llenan formatos de descripción de las estaciones base a utilizar en el levantamiento, plan de trabajo con la identificación de las bases por establecer, rutas y tiempos de traslado. Durante la medición se colectarán datos del levantamiento en una bitácora registrando: temperatura, estabilidad del terreno y condiciones meteorológicas.

El establecimiento de estaciones base deberá estar dentro de un rango de 40$60 \mathrm{~km}$ lo cual facilita una correcta densificación gravimétrica. El control de la red se efectuará ligándola al menos a dos estaciones base, previamente establecidas (estaciones de la red IGSN71). Deben usarse simultáneamente al menos dos gravímetros calibrados y, de ser posible, previamente verificados en un número de estaciones de la red IGSN71 o línea de calibración.

Las lecturas gravimétricas deberán ser simultáneas y las lecturas de tiempo tomadas con un solo reloj. Los circuitos estarán de tal modo que cierren sobre sí mismos observando todas las estaciones, para lo cual utilizarán el método conocido como de escalera. Todo circuito debe cerrarse en un tiempo menor de 24 horas y los circuitos empezarán y terminarán en las estaciones establecidas (véase Figura 12). 
En el caso de las estaciones gravimétricas de segundo orden, estas son estaciones sin marca física; los métodos de medición aplicados son mediante circuito o línea (véanse Figuras 13 y 14), todos partiendo de una estación base. El levantamiento debe completarse en un tiempo máximo de 72 horas.

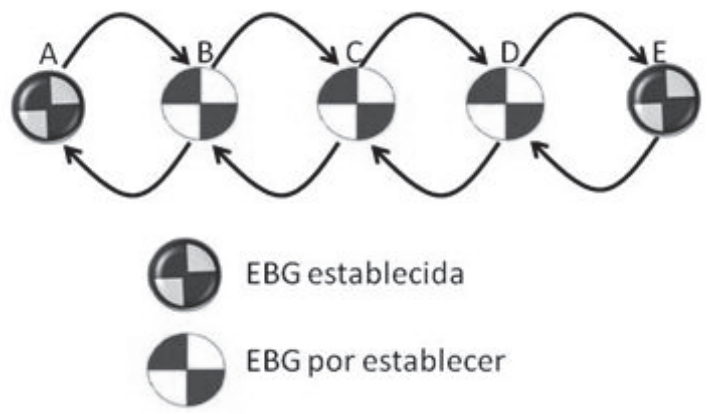

Figura 12. Método de escalera.

\section{Circuito:A1234A}

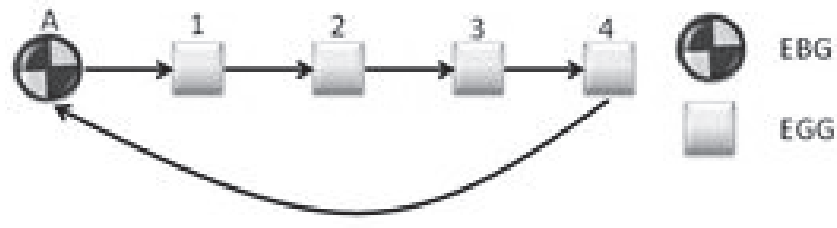

Figura 13. Método de circuito.

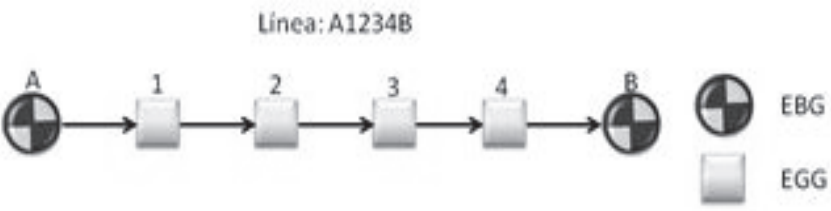

Figura 14. Método de línea.

\section{Procesamiento de los datos}

Se realiza por medio de ajuste de mínimos cuadrados. El procedimiento aprovecha la medición de redundancia para generar valores estadísticos que describen la congruencia de la medición. Como primer control de calidad se realiza la depuración de 
los datos de entrada realizando el análisis exploratorio de la congruencia, en la diferencia de los valores de aceleración de la gravedad medidos, y la diferencia de los valores de gravedad previamente definidos.

\section{Validación e integración de resultados}

Se realiza el análisis estadístico verificando que la desviación estándar de los valores de gravedad calculados permanezca dentro del rango admisible de 0.050 miliGales. En caso de obtener resultados fuera de este rango, se realiza una nueva revisión para ubicar la fuente del posible error no detectado en el procesamiento con apoyo del resumen de los datos estadísticos siguientes:

- Histograma de residuales

- Varianza de peso unitario

- Prueba de Chi cuadrada

- Detección de residuales elevados

Para cada EBG se debe llenar un formato que la describa y contenga todos los atributos necesarios conforme al Diccionario de Datos Geodésicos. En lo posible se monumenta o empotra una placa metálica.

\section{Conclusiones}

La generación y uso de datos geodésicos, bajo metodologías sustentadas, contribuyen al uso y aprovechamiento para generar insumos que den soporte a diversas actividades estadísticas y geográficas, como valor agregado al intercambio y la interoperabilidad de datos espaciales entre unidades productoras y usuarios nacionales e internacionales.

La aplicación de metodologías, métodos y procedimientos comprobados bajo Sistemas de Referencia y Estándares de Exactitud Posicional, la aplicación de diccionarios de datos, la elaboración de metadatos y la ejecución de pruebas de calidad que proveen información sobre los niveles de confiabilidad técnica de los datos para que el usuario determine la aptitud para su uso y aprovechamiento con base en sus necesidades (Gómora, 2014) generan certidumbre sobre la utilidad de los datos e información que sirve de insumo para la georreferenciación de objetos espaciales.

Los datos, productos y servicios geodésicos son capa fundamental de cualquier Infraestructura de Datos Espaciales; apoyan la georreferencia de temas de agua; emisiones, residuos y sustancias peligrosas; catastral y registral; cambio climático; Uso de Suelo, Vegetación y Recursos Forestales; Sector Energético, estudios de geodinámica terrestre, soporte geodésico a las instituciones encargadas de la defini- 
ción de límites municipales, estatales e internacionales, entre muchas aplicaciones más.

El campo de la geodesia está asociado inter, intra y multidisciplinariamente a la satisfacción de necesidades sociales, económicas, políticas, de conservación del medio ambiente y del aprovechamiento de recursos del espacio aéreo, terrestre y marino, entre otras.

Se debe considerar que los datos que aporta la Geodesia no son finitos, se conoce la dirección geográfica de un sitio de interés para un tiempo dado, más la Tierra se mueve y los fenómenos naturales pueden cambiar la geografía de un día para otro. Por lo tanto, esta ciencia contribuye al estudio de las variaciones de la superficie terrestre a través del tiempo, lo cual se cuantifica para evaluar los fenómenos dinámicos de nuestro planeta y ello requiere la producción continua de datos comparables, homogéneos y compatibles que sirvan de apoyo para el establecimiento de políticas públicas.

\section{Bibliografía}

Gómora, M., H.M. (2015). "La calidad como aptitud para el uso: evaluación de la exactitud posicional de los productos topográficos digitales escala 1:50,000 del INEGI", Revista Cartográfica, Instituto Panamericano de Geografía e Historia, núm. 91, enero-diciembre, pp. 159-174.

IERS (sin dato). General definitions and numerical standards. En IERS Technical Note 36, pp. 15-20. Recuperado de: <https://www.iers.org/SharedDocs/ Publikationen/EN/IERS/Publications/tn/TechnNote36/tn36_015.pdf?_blob= publicationFile\&v $=1>$

Instituto Nacional de Estadística y Geografía, INEGI (2010). Acuerdo por el que se aprueba la Norma Técnica para el Sistema Geodésico Nacional, en Diario Oficial. Recuperado de: <http://www.inegi.org.mx/geo/contenidos/normastecnicas/ doc/norma tecnica_para_el_sistema_geodesico_nacional.pdf $>$

(2015). Guía Metodológica de la Red Geodésica Horizontal, México. Re-

cuperado de: <http://www3.inegi.org.mx/sistemas/biblioteca/ficha.aspx?upc= 702825078805>

(2015). Guía Metodológica de la Red Geodésica Vertical, México. Recupe-

rado de: <http://www3.inegi.org.mx/sistemas/biblioteca/ficha.aspx? upc= $702825078782>$

(2015). Guía Metodológica de la Red Geodésica Gravimétrica, México.

Recuperado de: <http://www3.inegi.org.mx/sistemas/biblioteca/ficha.aspx? upc= 702825078799>

(2015). Geoide Gravimétrico Mexicano, México. Recuperado de: $<$ http://www.inegi.org.mx/geo/contenidos/geodesia/que_es_geoide.aspx 
International Terrestrial Reference Frame ITRF (2016) ITRF2008. Recuperado de: http://itrf.ign.fr/ITRF_solutions/2008/ $\geq$

Jet Propulsion Laboratory (sin dato) Grace Follow-On. Gravity Recovery and Climate Experiment Follow-On, en California Institute of Technology NASA, Recuperado de: <http://gracefo.jpl.nasa.gov/mission/>

Morelli, C. et al. (1972). The International Gravity Standardization Net 1971 (IGSN 71) Recuperado de: <http://www.dtic.mil/dtic/tr/fulltext/u2/ a006203.pdf>

Moritz, H. (2000). “Geodetic Reference System 1980”, J. Geod., 74(1):128-162. Recuperado de: <http://rd.springer.com/article/10.1007/s001900050278?noaccess $=$ true $>$

NOAA, National Geodetic Survey (2016) Vertical Datums. Recuperado de: $<$ http://www.ngs.noaa.gov/datums/vertical/> 